\title{
Direttori d'orchestra e maestri del coro anche se donne?
}

\author{
Paolo D'Achille
}

PUBBLICATO: 23 MARZO 2021

\section{Quesito:}

Ci sono pervenute (e continuano a pervenirci) molte domande sulle espressioni direttrice d'orchestra e maestra del coro: sono corrette o è preferibile usare i nomi maschili anche quando si parla di donne che svolgono tali professioni?

\section{Direttori d'orchestra e maestri del coro anche se donne?}

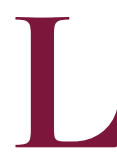

a questione di come denominare le donne che rivestono ruoli o svolgono professioni un tempo appannaggio esclusivo (o quasi) degli uomini è un tema che ricorre spesso sulle pagine dei giornali e nei dibattiti sui social, ogni tanto rinfocolati da qualche episodio mediatico o da certe dichiarazioni di personaggi pubblici (uomini o donne che siano), e probabilmente è destinata a durare ancora.

L'Accademia se ne è occupata diverse volte, nelle risposte pubblicate sulle pagine del servizio di Consulenza (vedi per esempio qui, qui e qui) e in varie altre occasioni (da ultimo, si veda qui l'equilibrato intervento di Vittorio Coletti tra i temi del mese e quil'articolo di Paola Villani su "Italiano digitale" a proposito del femminile di presidente). Torniamo a trattarne, "a grande richiesta" (come si dice nel mondo dello spettacolo), dopo che Beatrice Venezi ha preteso e ottenuto di essere presentata da Amadeus, al $7 \mathrm{I}^{\circ}$ Festival di Sanremo, come "direttore d'orchestra" e non come "direttrice d'orchestra" (fatto già commentato, in varie sedi, anche da autorevoli linguisti). Ma parleremo anche della scelta tra maestro del coro e maestra del coro e, più in generale, dell'uso di maestro o maestra come allocutivi, indirizzati appunto a chi opera in ambito musicale.

Iniziamo col dire che tanto direttrice quanto maestra sono nomi femminili attestati già nell'italiano antico: maestra (come si ricava dal corpus OVI) si documenta, a fine Duecento, nei Fiori e vita di filosafi e d'altri savi e dimperadori (I272-I275), in cui la storia è detta "maestra de la vita"; nel Libro dell'entrata e dell'uscita di una Compagnia mercantile senese degli anni I277-I282, che parla di I4 denari dovuti "a la maestra rimendatrice per rimendatura de li panni"; nel fiorentino Bono Giamboni, che nel suo Libro de' Vizî e delle Virtudi si rivolge più volte alla Filosofia personificata con l'allocutivo "Maestra de le (o delle) virtudi (o virtude)". Quanto a direttrice, nel senso di 'colei che dirige, che guida', si incontra (nella forma diritricie o dirittricie) in un testo degli anni I3I8-I320, il Reggimento e costumi di donna di Francesco da Barberino (cfr. la voce del TLIO).

Per venire a tempi a noi molto più vicini, nella scuola elementare postunitaria le maestre sono diventate ben presto più numerose dei maestri (e nella scuola per l'infanzia la maestra d'asilo, in passato detta maestra giardiniera, non ha praticamente mai avuto un corrispondente di sesso maschile). E nelle scuole elementari del passato non era neppure raro imbattersi nella direttrice didattica denominata come tale, sebbene in questo caso i direttori didattici siano stati, e a lungo, prevalenti, così come, del resto, $i$ presidi nelle scuole superiori e nelle facoltà universitarie. Ora si parla, per tutte le scuole primarie e secondarie, spesso inserite in "istituti comprensivi", di dirigenti scolastici e il problema del femminile si pone di nuovo, per la forma dell'aggettivo oltre che, come nel caso di preside, per l'articolo. 
Dunque, la questione non sta nella correttezza grammaticale delle forme maestra e direttrice (che è fuori discussione) ma nell'appropriatezza del loro uso in certe espressioni e in certi contesti. In effetti non di rado tra nomi maschili e corrispondenti nomi femminili si è avuto uno squilibrio e l'uso dei femminili è stato considerato improprio o inopportuno per indicare certe cariche o certe funzioni per molto tempo occupate quasi esclusivamente da uomini, tanto che alcune delle stesse donne che oggi le ricoprono percepiscono il femminile come una sorta di deminutio (a volte, effettivamente, è usato per tale scopo da giornalisti maschilisti) e preferiscono essere indicate con il maschile. Cosi segretaria è decisamente raro, nel campo politico e sindacale, come femminile di segretario di partito o segretario di un sindacato; lo stesso si può dire, in ambito universitario, per direttrice come femminile di direttore di dipartimento o di direttore generale, come pure per professoressa ordinaria invece di professore ordinario (si veda al riguardo l'articolo di Miriam Voghera e Debora Vena, Forma maschile, genere femminile: si presentano le donne, in Genere e linguaggio. I segni dell'uguaglianza e della diversità, a cura di Fabio Corbisiero, Pietro Maturi, Elisabetta Ruspini, Milano, FrancoAngeli, 2016, pp. 34-5I). Anche maestra ha un orizzonte d'uso più ristretto rispetto all'ampia gamma di significati propri di maestro: nel GRADIT il lemma maestra rinvia a maestro e qui si precisa che alcune delle numerose accezioni del maschile non prevedono l'uso del femminile.

Tuttavia, proprio in ambito musicale parliamo di maestra di musica, maestra di canto, maestra di pianoforte (una bella poesia di Marino Moretti si intitola La maestra di piano) e dunque non ci sarebbe nessun problema a usare maestra del coro o di coro. Eppure, perfino colei che in Italia è forse la più celebre rappresentante della professione, la compianta Mariele Ventre, indimenticata animatrice del piccolo coro dell'Antoniano di Bologna in tante edizioni del festival dello "Zecchino d'oro" (dal ig63 al I995), nella voce di Wikipedia viene definita "direttrice di coro".

Comunque, una ricerca in Internet con il motore Google in data I7 marzo 202I delle sequenze "maestra del coro" e "maestra di coro" restituisce rispettivamente circa 53.500 e 9.350 risultati (diversi dei quali sono riferiti proprio a Mariele Ventre). Con "direttrice d'orchestra" le cose vanno ancora meglio, perché i risultati sono circa I36.000.

Inoltre, Google libri ci permette di appurare che l'uso dei femminili non costituisce un'assoluta novità dell'italiano di oggi, nata in seguito alle rivendicazioni femministe. Di maestra del coro abbiamo addirittura due esempi settecenteschi: se la Istoria ecclesiastica di Giuseppe Agostino Orsi (vol. VI, Roma, Pagliarini, I750, pp. 407-408), quando dice che: "Gravemente commosso per un tal fatto l'Imperadore fece venire alla sua presenza la maestra del coro e senza rispetto alla sua canutezza e al suo venerabile aspetto comandò ad uno delle sue guardie di batterla in ambedue la guance fino ad imbrattarle tutta la faccia di sangue", si riferisce a un fatto della storia antica ed è dunque poco significativo, l'espressione compare in corsivo, con riferimento all'attualità, in un testo in inglese di alcuni anni prima, The Present State of Music in France and Italy di Charles Burney (London, Beckett, I72I, p. I83): "the first violin was very well played by Antonia Cubli, of Greek extraction; the harpsichord sometimes by Francesca Rosli, maestra del coro, and sometimes by others; these young persons frequently change instruments". Non mancano neppure esempi dell'Ottocento né, ovviamente, del secolo scorso.

Di direttrice d'orchestra, dopo aver rimandato al volume Storia di una "novita": la direzione d'orchestra al femminile. Atti della giornata internazionale di studi, Firenze, I9 giugno 2003 (Firenze, Consiglio regionale della Toscana, Commissione regionale pari opportunita donna-uomo, 2004), all'interno del quale l'espressione è usata anche al plurale, possiamo segnalare come più antico esempio reperito in Google libri una notizia data sul periodico "Il Pirata. Giornale di Letteratura, Belle Arti e Teatri" (XVI, I8 gennaio I851, n. 58, p. 232: "Il Correo di Madrid dà per certo che la Frezzolini fu nominata 
direttrice di scena a quel R. Teatro d'Oriente, e direttrice d'Orchestra"), a cui, nel XIX secolo, fanno seguito altre attestazioni. Passando al primo Novecento, rileviamo che nel Censimento della popolazione del Regno d'Italia al 10 febbraio 1901 (vol. II, Roma, Tipografia Nazionale di G. Berterio, 1903, p. 530 nota a) è "Compresa I direttrice d'orchestra". Sui periodici dell'epoca si leggono passi come: "Una donna direttrice d'orchestra nell'attuale stagione lirica al Politeama di Livorno, è la signorina Palmira Orso" ("Musica e musicisti. Rivista illustrata bimestrale", vol. 6o, n. I, gennaio 1905, p. 58) e "La maestra di musica signora Steiner di Hamburg è nominata concertatrice e direttrice dorchestra nella Sala dei Concerti a Dresda" ("Ars et Labor. Musica e musicisti", vol. 6I, n. I, gennaio I9o6, p. 278). Anche il "Corriere dei Piccoli" del 3r maggio r9I3, in un interessante articolo intitolato "Dove prima erano gli uomini" (p. I3), riporta tre volte l'espressione, in un caso nella didascalia sotto la fotografia della "Signorina Kitschmann, direttrice d'orchestra diplomata". Infine, parla di "direttrice d'orchestra" la voce fuori campo di un filmato dell'Istituto LUCE ("La Settimana Incom" oro7o del I8/3/1954) dedicato a Carmen Bulgarelli Campori e l'espressione figura anche nei titoli di un paio di giornali che compaiono nel corso del filmato, che però è intitolato "Direttrice in gonnella" (ci si sarebbe se mai aspettato Direttore...).

Dunque, anche il retroterra storico legittima l'uso del femminile, che va certamente consigliato (e invitiamo senz'altro a farlo), ma che tuttavia non può essere imposto a chi, come Beatrice Venezi, lo ritiene (sia pure a torto) riduttivo e preferisce essere indicata con la forma maschile. Non è vero che il maschile sia, come lei ha detto, "il nome preciso" della sua professione; tuttavia, a sua attenuante, possiamo dire che nel mondo della musica si usano i termini soprano e contralto ancora prevalentemente al maschile pur indicando donne e sono frequenti metonimie come primo violino, primo flauto, anche quando a suonare questi strumenti sono delle signore. Ciò può forse spiegare il suo errore. Del resto, anche in un testo recente, scritto da una donna, si legge quanto segue:

Il primo ostacolo sembra essere di natura linguistica, perché non esiste la parola adatta per indicare i direttori d'orchestra donne; a chiamarle "direttrice" viene in mente una signora austera, che striglia gli studenti nei corridoi di un collegio; mentre il nome "maestra" richiama la scuola elementare.

È dunque meglio dire il Maestro Speranza Scappucci, il Direttore d'orchestra Beatrice Venezi (per citare due fra le italiane che si cimentano con successo nella direzione)? (Anna Rollando, Applaudire con i piedi. Segreti e curiositá della musica colta, Roma, Graphofeel 2018, e-book)

Google libri fornisce anche vari esempi in cui direttore d'orchestra è detto di donne. Ne cito un paio, riferiti a due note direttrici americane:

Sarah Caldwell è una delle pochissime donne direttore d'orchestra; guida lei il divertente Don Pasquale della Emi. ("Oggi", 35, 1979, [manca l'indicazione del fascicolo], p. 35; ritengo improbabile interpretare qui direttore come plurale di direttora, forma peraltro preferita a direttrice da molte femministe)

Nel I974 se n'è avuta un'secuzione in forma di concerto a New York con protagonista Montserrat Caballé, che, sempre in associazione con il direttore d'orchestra Eve Queler, ha successivamente riproposto Parisina sulla scena: a Nizza e Barcellona nel dicembre 1977. (William Ashbrook, Donizetti. La vita, Torino, EDT, I986, p. 70; non sarà un caso che si tratti di una traduzione dall'inglese)

Ci sarebbe anche un esempio precedente: il Fondo Vedo dell'Archivio dell'Istituto LUCE conserva infatti varie fotografie del "Concerto della giovanissima Giannella De Marco direttore d'orchestra" tenuto al Teatro delle Vittorie il I8 novembre del I957, ma non si può essere certi che il titolo sia coevo all'evento. Lo stesso vale per la descrizione di un altro filmato dell'Istituto LUCE, intitolato Un documento prezioso ("La Settimana Incom" o2344 del I4/3/I963) dedicato alla stessa giovane musicista, in cui si usa invece il femminile (coerentemente col successivo pittrice): "Intervista a Giannella De 
Marco, direttrice d'orchestra e pittrice a I9 anni" (nel corso del filmato, molto ironico, la voce fuori campo non usa né il maschile né il femminile, limitandosi a dire che a quattro anni e mezzo la bambina prodigio "per la prima volta sali sul podio del direttore d'orchestra").

Per la direzione del coro, invece, ho trovato in Google solo rarissimi e recentissimi esempi di maestro di (o del) coro donna:

Sono state poi invitate dalla presidente a relazionare in merito all'attività lavorativa delle socie Maria Luisa Cordone ed Erina Gambarini, l'una esperta di comunicazione e sviluppo reti di business, l'altra unico maestro di coro donna in Italia. ("Club Bergamo. Conviviale relazioni dei gruppi di lavoro", Soroptimist.it, II/II/20I4)

«La storia di questo teatro, dall'estetica e dall'acustica uniche, sconfina nella leggenda e l'emozione rapisce. Ma al piacere consentitemi di aggiungere un piccolo vezzo: sono il primo Maestro del Coro donna dal I737!». A parlare è Gea Garatti Ansini, appena nominata Maestro del Coro del Teatro San Carlo. (Dario Ascoli, San Carlo, Gea Garatti Ansini prima donna alla guida del Coro. Il maestro bolognese subentra a Marco Faelli. Domani il primo impegno, "Corriere della Sera. Corriere del Mezzogiorno. Cultura", I/9/2018)

Il problema del femminile si pone piuttosto per l'allocutivo: rivolgersi a una musicista (direttrice d'orchestra, maestra del coro, flautista, soprano) chiamandola maestra, forma perfettamente parallela al maestro che viene usato per gli uomini (e che in passato è stato indirizzato, per esempio, a Claudio Abbado, Franco Potenza, Severino Gazzelloni, Luciano Pavarotti, ma anche a registi come Giorgio Strehler e Luca Ronconi; e va ricordato che maestro è allocutivo/appellativo anche per gli chef), sarebbe del tutto logico. Tuttavia, effettivamente, non ci pare che si tratti di un uso consolidato (e non ci risulta che in passato siano state interpellate con il titolo di maestra neppure dive come Maria Callas e Renata Tebaldi). Che il problema sia avvertito da tempo è documentato da questo brano di un articolo di Paola Fallaci (una donna, dunque!), dedicato ancora a Giannella De Marco, pubblicato su un fascicolo del volume 43 di "Oggi" del 1987 (p. 64, che fornisce un ulteriore esempio di direttrice d'orchestra):

Roma, gennaio. Sul taxi che mi portava da Giannella De Marco, direttrice d'orchestra, seguitavo a chiedermi: come mi posso rivolgere a una donna che fa quel mestiere? Le dico: «Buongiorno maestra»? Per carità. Le dico: «Buongiorno direttrice»? Macché. Forse va bene «Buongiorno signora».

C'è da augurarsi che il femminile maestra si faccia largo anche in ambito musicale, e non ci sono ragioni perché non si possa affermare. Di certo a bloccarne l'uso sta il fatto (richiamato anche nel passo di Anna Rollando sopra riportato) che per maestra si intende prevalentemente l'insegnante delle scuole elementari (campo in cui, come si è detto allinizio, il genere femminile è prevalente) e che quindi l'uso allocutivo del termine sia proprio soprattutto dell'età infantile; così il suo impiego da parte di persone adulte che devono rivolgersi a musiciste (a volte più giovani di loro) sembra creare imbarazzo sia a chi lo dà sia a chi lo riceve. Ma non dovrebbe essere così gli esempi duecenteschi citati all'inizio ci ricordano che anche la storia e la filosofia (quest'ultima personificata) erano considerate maestre. E comunque anche il possibile richiamo alla maestra elementare dovrebbe suonare tutt'altro che offensivo: per molte persone la maestra delle scuole elementari resta tuttora la figura più autorevole (e cara) tra tutti i docenti, maschi e femmine, che hanno incontrato nel proprio percorso di studi. È certamente lei che ci ha fornito le nozioni fondamentali, su cui si appoggiano tutte le altre, e che ci ha insegnato le cose che abbiamo imparato davvero, tanto da non dimenticarle (e da non dimenticarla) più. 


\section{Cita come:}

Paolo D'Achille, Direttori d'orchestra e maestri del coro anche se donne? , "Italiano digitale", 2021, XVI, 2021/1 (gennaio-marzo)

DOI: $10.35948 / 2532-9006 / 2021.6496$

Copyright 2021 Accademia della Crusca

Pubblicato con licenza creative commons CC BY-NC-ND 